\title{
SHARED CIRCULAR RADIATOR FOR UWB-MIMO APPLICATIONS
}

\author{
K. SRIVIDHYA ${ }^{1} \&$ H. UMMA HABIBA ${ }^{2}$ \\ ${ }^{1}$ Research Scholar, Anna University, Chennai, Tamil Nadu, India \\ ${ }^{2}$ Professor, Sri Venkateswara College of Engineering, Chennai, Tamil Nadu, India
}

\begin{abstract}
A compact dual-polarized lower ultra-wideband multi-input multi-output antenna made of the shared radiating element is proposed. The antenna is a microstrip line fed and has a dimension $19.5 \times 19.5 \times 0.787 \mathrm{~mm} 3$ with the partial ground plane. Slots are etched in the radiator and the ground, which helps reducing mutual coupling thereby increasing MIMO performance. Miniaturization is achieved using the partial ground plane. The antenna shows good performance covering a bandwidth of around $11 \mathrm{GHz}$ in the ultra-wideband spectrum catering to the requirements of WLAN, WWAN, WiMax, WiFi, LTE-A, ISM, USB dongle applications to mention the important ones. The designed antenna is modeled and simulated using Advanced Design System simulator.

KEYWORDS: Multiple Input Multiple Output (MIMO) Antenna, Shared Radiator \& Ultra Wide Band (UWB) Communications
\end{abstract}

Received: Jul 17, 2018; Accepted: Aug 10, 2018; Published: Aug 23, 2018; Paper Id.: IJCNWMCOCT20181

\section{INTRODUCTION}

Modern communication systems demand, a huge volume of data to be transferred at high speed and accuracy without altering the power limitations of the system. UWB technology has attracted more attention from the Federal Communication Commission (FCC) released the band for commercial systems. Moreover, it exhibits many attractive features like low interference, low power density, low cost, low complexity and very high data rate. Also, the antenna exhibits simple structure rendering it much suitable for wireless applications. Hence the design of an omnidirectional antenna with gain flatness and linear phase takes precedence. To mitigate multipath fading, MIMO is proposed. MIMO combines the bounced and reflected streams of data arriving at different time intervals at the receiver reducing the chance of signal degradation. Allowing for greater quantity and quality of data over the network, overall throughput is maximized. MIMO technology utilizes multiple antenna elements at the transmitter and receiver to have significant improvement in the capacity. Research is oriented towards placement of antenna elements to fit the small space in handheld terminals. This lightweight, compact, easy to fabricate antenna elements with low correlation needs to be developed for MIMO applications.

Various effective decoupling methods are proposed in the literature to reduce mutual coupling between the elements. Diversity schemes are employed [Jian Ren. et. al, 2014, Toktas, 2016, Ghouz.et.al, 2015], they act like dual polarised antennas exhibiting perpendicular gain patterns. Neutralization lines [Peng Liu. et. al, 2018, Wang. et. al, 2014] provide an additional coupling path to offset the coupling. Electromagnetic bandgap structures [Zhang. et. al, 2015] inhibit the propagation of surface waves thereby providing isolation. Defected ground structures [Nandi. et. al, 2017] act as band stop filters to achieve high isolation. Lumped network [Li.et.al, 2015] provide additional 
parasitic effects to cancel the coupling. Resonator decoupling network [Zhao.et.al, 2014] suppresses surface waves, achieving isolation. Meander line decoupling [Hari.et.al, 2015] stops surface waves creating a strong magnetic coupling. Usage of stubs and slots are discussed in [Mao.et.al, 2014]. Often shared radiators that achieve high compactness is discussed in the literature [khan. et. al, 2015, Patre. et. al, 2018]. A printed shared circular disc planar MIMO antenna is selected for discussion in this paper due to its compact size and wide applicability in UWB [Sharma.et.al, 2017, Ritesh.et.al, 2016]. The system works on dual polarization with a dimension of $19.5 \times 19.5 \times 0.787 \mathrm{~mm} 3$ with improved radiation pattern and stable gain. Isolation between the elements is achieved by virtue of polarization diversity as well as slots on the radiator, for operation over UWB band where plenty of wireless applications are crowded. The antenna exhibits better compactness among its similar counterparts without any added complexity in structure.

The remainder of the paper is organized as follows. The next section describes the design and evolution of the antenna. Subsequent Section discusses the performance and far-field radiation pattern generated by a single circular patch antenna with two separated feeds so that orthogonal radiation patterns can be generated. Then we show the calculation of correlation coefficients and diversity performance. Conclusions are offered at the end.

\section{SHARED CIRCULAR RADIATOR DESIGN}

The shared radiating element is circular in shape, fed by two microstrip line designated as port 1 and 2. Dual polarisation is achieved by orienting the feeds perpendicular to each other. The circular monopole antenna is printed on a readily available and low cost substrate FR4 with relative permittivity $\varepsilon_{\mathrm{r}}=4.4$ and loss tangent 0.025 with thickness $h=$ $0.787 \mathrm{~mm}$. Compared against the conventional MIMO systems, shared radiator can achieve greater compactness[Moradi.et.al, 2014, zhao.et.al, 2016]. The defected ground plane enhances the impedance matching of the antenna. Element decoupling is achieved by etching a rectangular slot in the radiator at cross angles to each other. A slot is also etched on the ground plane for better results. The overall size of the antenna is $19.5 \times 19.5 \times 0.787 \mathrm{~mm}^{3}$. The patch is designed, keeping in mind that with the increase in radius, the resonance gets shifted towards the lower frequency.

$$
\mathrm{LF}=\frac{7.2}{2.25 * \mathrm{R}+\mathrm{g}}
$$

where $\mathrm{R}$ is the radius of the disc and $\mathrm{g}$ is the gap height. The evolution and geometry of the proposed antenna are shown in Figure 1 (a-c). The final geometry of the proposed antenna is in Figure 2.

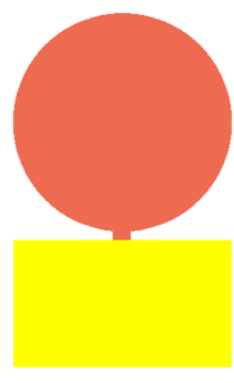

(a) Antenna

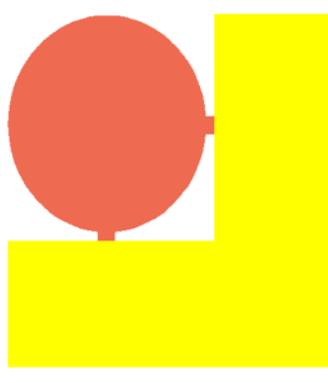

(b) Antenna

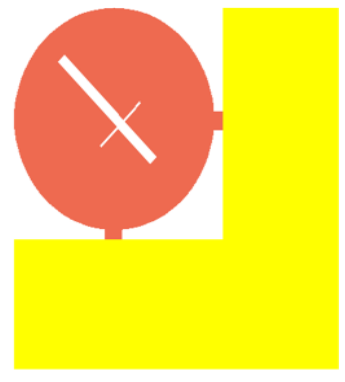

(c) Antenna

Figure 1: Evolution of the Shared Circular Radiator Antenna 

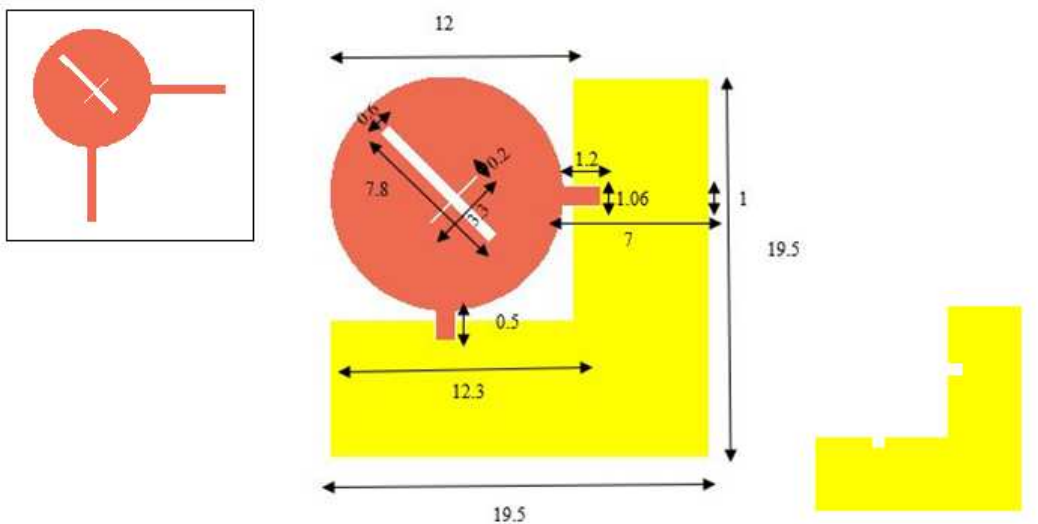

Figure 2: Proposed Shared Circular Radiator Antenna- Geometry

\section{RESULTS AND DISCUSSIONS}

This section presents a discussion on the results obtained by simulations carried out on a commercial ADS software. The performance of the proposed antenna is evaluated.

The proposed shared circular radiator is modeled as per the designed dimensions and the layout is shown in Figure 3. A circular antenna can be thought of as an infinite set of dipoles fed from a single point offering low dispersion characteristics. It is demonstrated through experiments and analysis, that a circular monopole antenna has ten times octave impedance bandwidth and constant group delay behavior. Furthermore, with smooth fluctuations of the real part about the mean of 50ohms, and the imaginary part around $0 \mathrm{ohms,} \mathrm{it} \mathrm{exhibits} \mathrm{good} \mathrm{matching} \mathrm{with} \mathrm{50ohms} \mathrm{feed.} \mathrm{Due} \mathrm{to} \mathrm{larger}$ bandwidth, it is required that the antenna has low dispersion characteristics throughout UWB. The frequency domain characteristics are dependent on the feed gap, ground plane, and disc dimensions.

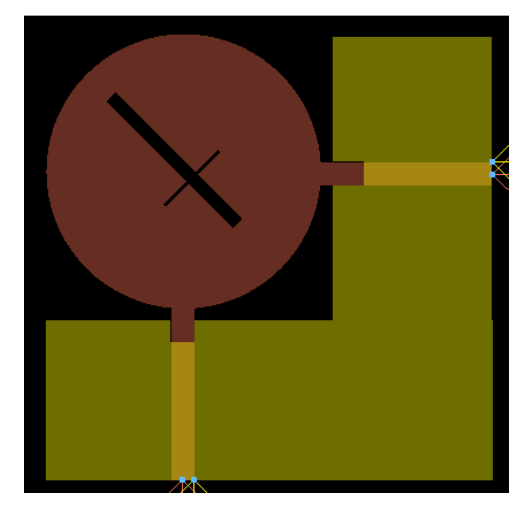

Figure 3: Proposed Antenna Layout

The input impedance and in turn the impedance bandwidth is dictated by the feed gap. Cross polar components occur at the upper edge of the ground plane near the radiating patch due to the excitation of higher order modes. Cross polarization is because of the discontinuity at the substrate - air and patch - dielectric interface resulting in surface waves which are minimized by reducing dielectric constant and dielectric thickness. Considering high-frequency solution in terms of geometric optics and ray diffractions, for the horizontal electric dipole upon a truncated, semi-infinite grounded dielectric slab, we have contributions from space waves, surface waves and leaky waves[Maci.et.al,1996]. Thus there occurs a shift in resonant frequency due to the coupling of radiating magnetic currents and ground plane edges given by 


$$
\frac{\Delta \mathrm{f}}{\mathrm{f}}=-4 \sqrt{\varepsilon_{\mathrm{e}}} \frac{\Delta \mathrm{I}_{\mathrm{d}}}{\lambda_{0}}
$$

Where $\Delta \mathrm{I}_{\mathrm{d}}$ is the length extension due to diffraction. Slots were etched on the radiator, forcing the RF current to remain concentrated on the edges of the slots instead of traveling from one port to the other and help reduce mutual coupling. A defected ground structure is thought of as periodic or nonperiodic slots or defects on the ground plane of planar microwave circuits. A 'defect' so placed, perturbs the shield current distribution and is considered as an approximation of an infinite, perfect-conducting current sink. The fundamental unit of DGS is a gap or slot in the ground metal, aligned for efficient coupling. The equivalent circuit seems to be a parallel-tuned circuit in series with the transmission line to which it is coupled [Breed, 2018]. In addition, a small rectangular slot is also cut out, to improve antenna electrical performance. The variation in reflection coefficient with frequency is shown in Figure 4. From the plot, it could be found that the shared radiator resonates for the entire UWB band. The 10db impedance bandwidth is observed from $2-13 \mathrm{GHz}$. The input return loss as calculated from the plot is $-28 \mathrm{~dB}$ and output return loss are $-26 \mathrm{db}$ on an average. The isolation factor is observed to vary from $26 \mathrm{db}$ in the lower band to $23 \mathrm{db}$ in the upper band. It is observed that the proposed antenna shows a stable radiation pattern all over the UWB band as observed from the following 3D and 2D plot in Figure 5(a-b) and 6(a-b).

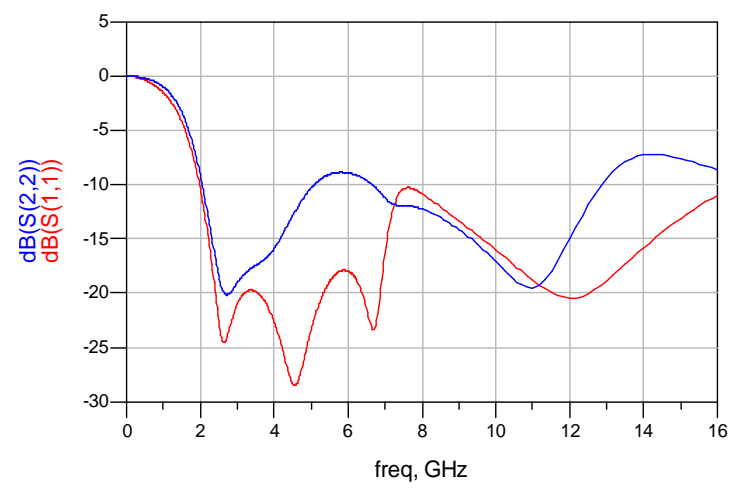

Figure 4(a): Reflection Coefficient Plot

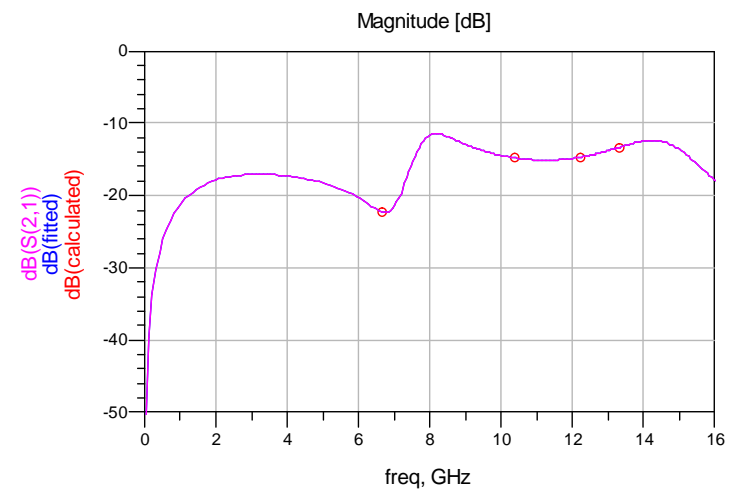

Figure 4(b): Coupling Coefficient Plot

It is observed that the proposed antenna shows a stable radiation pattern all over the UWB band as observed from the following 3D and 2D plot in Figure 5(a-b) and $6(a-b)$.

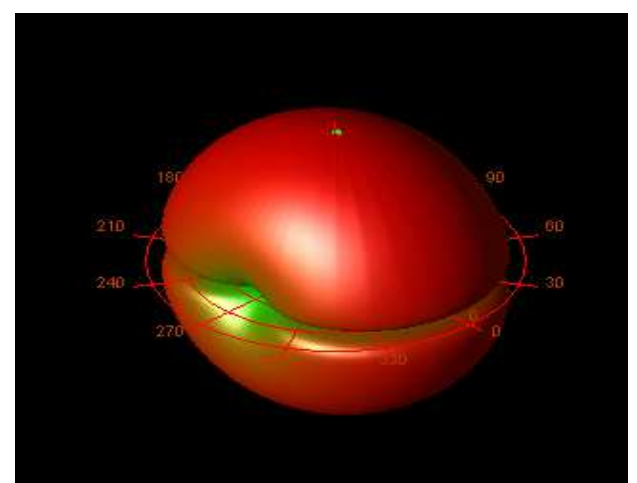

Figure 5(a): 3D Plot of Radiation

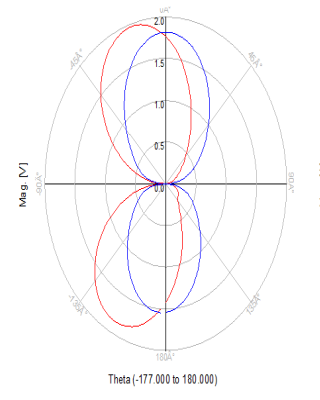

E theta E phi

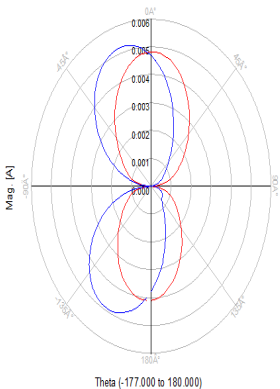

H theta $\mathrm{H}$ phi

Figure 5(b): 2D Plot of Radiation Pattern Pattern in Lower UWB Band 


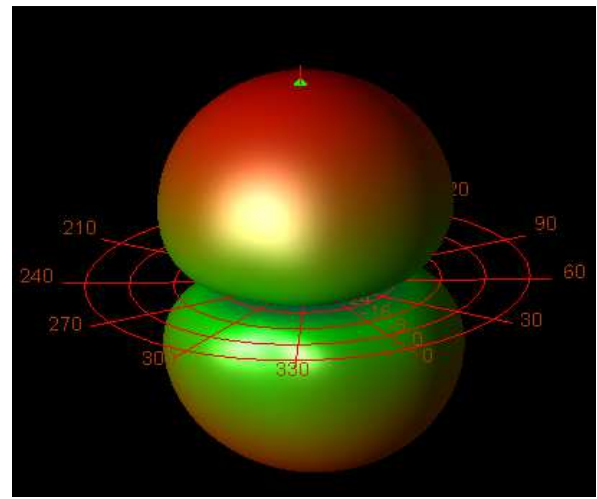

Figure 6(a): 3D Plot of Radiation

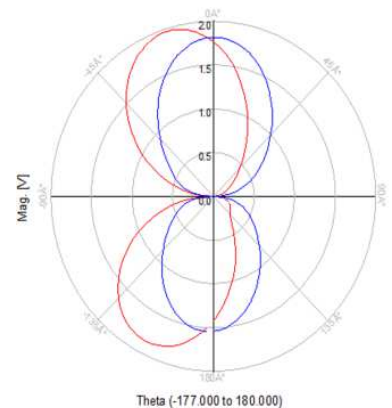

E theta E phi

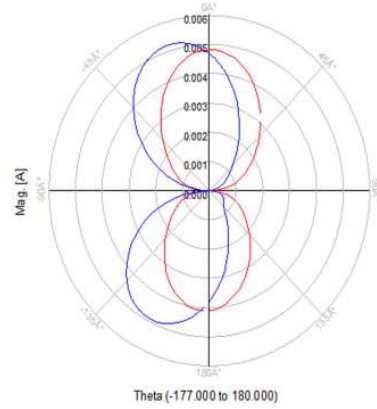

H theta H phi

Figure 6(b):2D Plot of Radiation Pattern in Higher UWB Band

The gain and directivity values are observed to vary from $2.2 \mathrm{dbi}$ to $6.9 \mathrm{dbi}$ as we proceed from lower to higher frequencies. The plot is as shown in Figure 7 (a-b). The radiation efficiency varies from $85 \%$ to $98 \%$ much satisfying the requirement of UWB antennas.

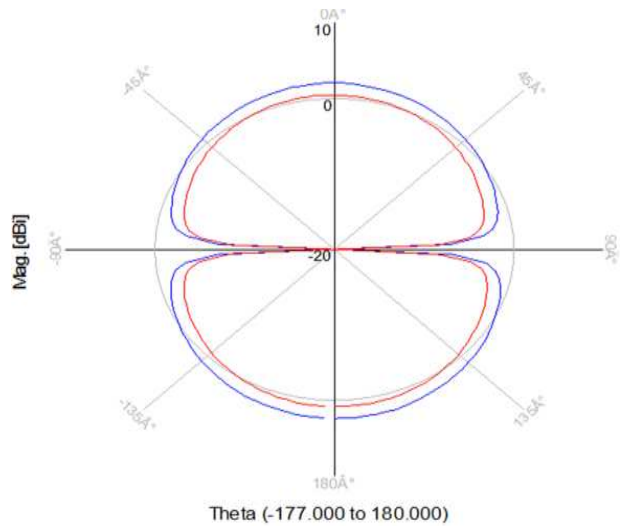

Directivity

Gain

Figure 7(a): Gain and Directivity in Lower Band

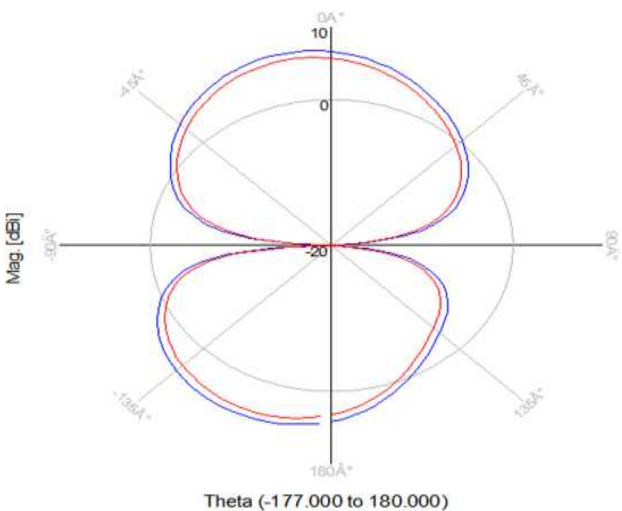

Directivity Gain Higher Band

\section{MIMO PERFORMANCE}

It is always mandatory to evaluate the performance of the proposed antenna as per the requirements of MIMO technology. Figure 8 (a-d) shows the plot of MIMO parameters from which it is evident that the proposed antenna satisfies low correlation and maintains good diversity performance.

Envelope Correlation Coefficient tells us how independent are the radiation patterns, polarisation and phase of the fields between two antennas and is given by

$$
\rho=\frac{\left|S 11^{*} S 12+S 21^{*} S 22\right|^{2}}{\left(1-|S 11|^{2}-|S 21|^{2}\right)-\left(1-|S 22|^{2}-|S 12|^{2}\right)}
$$

The plot of ECC in Figure 8(a) shows a minimal correlation of 0.02 in the required band.

Diversity gain is the decreased required receive SNR for a given bit error rate (BER) averaged over the fading. 
Div Gain=10 $(1-\rho)^{0.5}$

The plot of diversity gain in Figure 8(b) shows a value of $9.8 \mathrm{db}$.

Mean effective gain (MEG), quantifies the link power in multipath channels.

$\mathrm{MEG}_{\mathrm{i}}=$ Total Eff $_{\mathrm{i}} / 2$,

Where total efficiency consists of mismatch efficiency and radiation efficiency.

The plot of MEG in Figure 8(c) shows a value of -4db over the band.

Group delay is the time delay of the amplitude envelopes of the various frequency components of a signal through the channel.

Group delay $=-\Delta \varphi / \Delta \omega$

The plot of MEG in Figure 8(d) shows a constant behavior over the band.

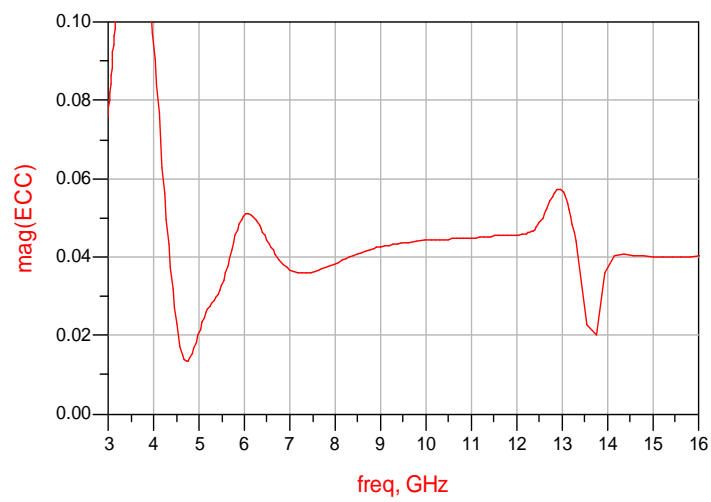

Figure 8(a): Envelope Correlation Coefficient

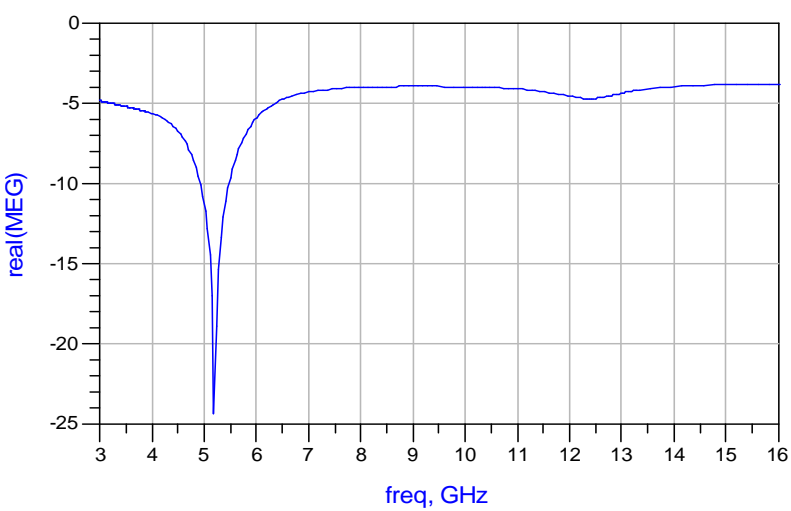

Figure 8(c): Mean Effective Gain

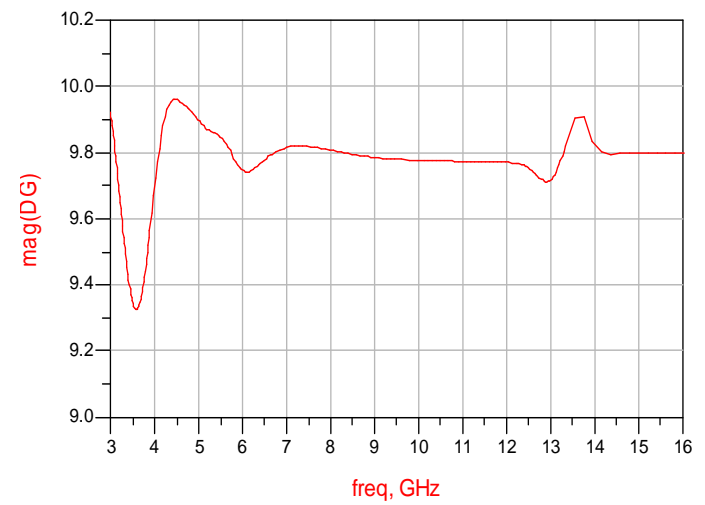

Figure 8(b): Diversity Gain

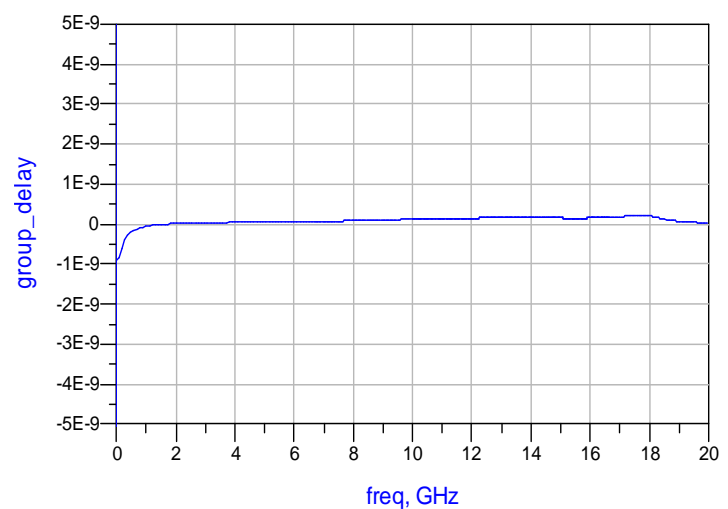

Figure 8(d): Group Delay

A comparison of the dimension and performance of the various antenna using shared radiator is listed in the table 1 in the below. 
Table 1: Comparison of Antenna Performance Using Shared Radiator

\begin{tabular}{|c|c|c|c|c|}
\hline $\begin{array}{l}\text { Author } \\
\text { (Year) }\end{array}$ & Type of antenna & Measurements & Design Parameters & Application \\
\hline $\begin{array}{l}\text { Situ Rani Patre } \\
(2018)[16]\end{array}$ & $\begin{array}{l}\text { Leaf shaped } \\
\text { shared radiator }\end{array}$ & Gain: $5.22 \mathrm{dbi}$ & 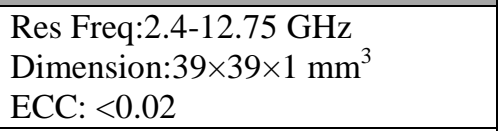 & $\begin{array}{l}\text { UWB } \\
\text { applications }\end{array}$ \\
\hline $\begin{array}{l}\text { Saeed Khan (2017) } \\
\text { [15] }\end{array}$ & $\begin{array}{l}\text { Modified patch } \\
\text { common radiator }\end{array}$ & Gain: 4 dbi & $\begin{array}{l}\text { Res Freq: } 3-10.6 \mathrm{GHz} \\
\text { Dimension: } 22 \times 24.3 \times 1 \mathrm{~mm}^{3} \\
\text { ECC: }<0.42\end{array}$ & $\begin{array}{l}\text { UWB } \\
\text { applications }\end{array}$ \\
\hline $\begin{array}{l}\text { Abubeker A. } \\
\text { Yussuf(2017) } \\
{[21]}\end{array}$ & $\begin{array}{l}\text { Hexagonal } \\
\text { common radiator }\end{array}$ & Gain: $5.5 \mathrm{dbi}$ & $\begin{array}{l}\text { Res Freq:2-3GHz } \\
\text { BW: } 1 \mathrm{GHz} \\
\text { Dimension: } 108 \times 108 \times 1.6 \mathrm{~mm}^{3} \\
\text { ECC: } 0.3\end{array}$ & $\begin{array}{l}\text { LTE, } \\
\text { WiFi }\end{array}$ \\
\hline $\begin{array}{l}\text { Nan Zhao (2016) } \\
{[12]}\end{array}$ & $\begin{array}{l}\text { Trapezoidal } \\
\text { shared radiator }\end{array}$ & Gain: $2.3 \mathrm{dbi}$ & 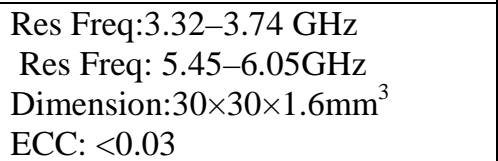 & $\begin{array}{l}\text { WiMax/ } \\
3.5 \mathrm{G} \\
\text { WLAN/ } \\
5.8 \mathrm{G}\end{array}$ \\
\hline $\begin{array}{l}\text { Chun-Xu Mao } \\
(2014)[14]\end{array}$ & $\begin{array}{l}\text { Pentagonal } \\
\text { coradiator }\end{array}$ & Gain: 4 dbi & $\begin{array}{l}\text { Res Freq: } 3-11 \mathrm{GHz} \\
\text { Dimension: } 40 \times 40 \times 0.8 \mathrm{~mm}^{3} \\
\text { ECC: }<0.05\end{array}$ & $\begin{array}{l}\text { UWB } \\
\text { applications }\end{array}$ \\
\hline Proposed & $\begin{array}{l}\text { Shared Circular } \\
\text { Radiator }\end{array}$ & Gain: 6.9dbi & $\begin{array}{l}\text { Res Freq: } 2-13 \mathrm{GHz} \\
\text { BW: } 11 \mathrm{GHz} \\
\text { Dimension: } 19.5 \times 19.5 \times 0.787 \mathrm{~mm}^{3} \\
\text { ECC: } 0.02\end{array}$ & $\begin{array}{l}\text { UWB } \\
\text { wireless } \\
\text { applications }\end{array}$ \\
\hline
\end{tabular}

\section{CONCLUSIONS}

- An ultra-compact MIMO antenna of overall dimension of $19.5 \times 19.5 \times 0.787 \mathrm{~mm}^{3}$, with a single, shared radiator fed in dual polarisation using microstrip line resonating for the UWB spectrum is proposed.

- Miniaturisation is achieved using defected ground and antenna shows low mutual coupling due to slots on the radiator.

- The antenna exhibits favourable radiation behaviour throughout the band and exhibits a stable gain of 4-7dbi. The system exhibits excellent MIMO performance, thus rendering it a suitable candidate for varied wireless applications enhancing capacity.

\section{REFERENCES}

1. Jian Ren, Dawei Mi, and Yingzeng Yin, "Compact Ultra wideband MIMO Antenna with WLAN/UWB Bands Coverage ', Progress In Electromagnetics Research C, Vol. 50, 121-129, 2014.

2. Peng Liu, Deming Sun, Peng Wang and Peng Gao, "Design of a Dual-Band MIMO Antenna with High Isolation for WLAN Applications", Progress In Electromagnetics Research Letters, Vol. 74, 23-30, 2018.

3. Xiaoyan Zhang, Xinxing Zhon, Bincheng Li, Yiqiang Yu,. "A Dual-Polarized MIMO Antenna with EBG for 5.8GHz WLAN Application", Progress In Electromagnetics Research Letters, Vol. 51, 15-20, 2015.

4. Wang, Y. and Z.-W. Du, "A wideband printed dual-antenna with three neutralization lines for mobile terminals" IEEE Trans. Antennas Propag., Vol. 62, No. 3, 1495-1499, Mar. 2014.

5. Nandi, S. and A. Mohan, “A compact dual-band MIMO slot antenna for WLAN applications," IEEE Antennas Wirel. Propag. Lett., Vol. 16, 2457-2460, 2017. 
6. Abdurrahim Toktas,"G-shaped band-notched ultra-wideband MIMO antenna system for mobile terminals", IET Microwave Antennas and Propagation., 2016, Vol. 11 Issue. 5, pp. 718-725.

7. Hussein Hamed Mahmoud Ghouz," Novel Compact and Dual-Broadband Microstrip MIMO Antennas for Wireless Applications', Progress In Electromagnetics Research B, Vol.63, 107-121, 2015

8. Hari S. Singh, Gaurav K. Pandey, Pradutt K. Bharti, Manoj K. Meshram, "Compact Printed Diversity Antenna for LTE700/GSM1700/1800/UMTS/Wi-Fi/Bluetooth/LTE2300/2500 Applications for Slim Mobile Handsets", Progress In Electromagnetics Research C, Vol. 56, 83-91, 2015.

9. Li, R. P., P. Wang, Q. Zheng, and R. Z. Wu, "Compact microstrip decoupling and matching network for two symmetric antennas," Electron. Lett., Vol. 51, No. 18, 1396-1398, 2015.

10. Zhao, L., L. K. Yeung, and $K .-L$. Wu, "A coupled resonator decoupling network for two-element compact antenna arrays in mobile terminals," IEEE Trans. Antennas Propag., Vol. 62, No. 5, 482-289, 2014.

11. Alishir Moradi, Kordalivand, Tharek A. Rahman, Mohsen Khalily, "Common Elements Wideband MIMO Antenna System for WiFi/LTE Access-Point Applications", IEEE Antennas And Wireless Propagation Letters, VOL. 13, 2014.

12. Nan Zhao, Wen-Peng Tian, "CPW-Fed Dual-Band MIMO Antenna with Common Radiating Element", Progress In Electromagnetics Research Letters, Vol. 62, 71-75, 2016.

13. Hashemi, S., \& Kazemian, B. (2014). A Dialogical Nature of Structure in Keats's Odes as a Circular Escape from Pain to Pleasure: A Bakhtinian Perspective.

14. Chun-Xu Mao and Qing-Xin Chu, "Compact Coradiator UWB-MIMO Antenna With Dual Polarization", IEEE Transactions on Antennas and Propagation, vol. 62, no. 9, September 2014.

15. Muhammad Saeed Khan, Antonio-Daniele Capobianco, Adnan Iftikhar, Raed M. Shubair, Dimitris E. Anagnosto, Benjamin D. Braaten,"Ultra-compact dual-polarised UWB MIMO", IET Microw. Antennas Propag., 2017, Vol. 11 Iss. 7, pp. $997-1002$.

16. Situ Rani Patre, Surya P. Singh, "Shared radiator MIMO antenna for broadband applications" IET Microwave, Antennas and Propagation, 2018, Vol. 12 Iss. 7, pp. 1153-1159.

17. Manish Sharma, Yogendra K. Awasthi, Himanshu Singh," Design of CPW-Fed High Rejection Triple Band-Notch UWB Antenna on Silicon with Diverse Wireless Applications", Progress In Electromagnetics Research C, Vol. 74, 19-30, 2017.

18. Ritesh Kumar Saraswat, Mithilesh Kumar," Miniaturized Slotted Ground UWB Antenna Loaded with Metamaterial for WLAN and WiMAX Applications", Progress In Electromagnetics Research B, Vol. 65, 65-80, 2016.

19. Gary Breed," An Introduction to Defected Ground Structures in Microstrip Circuits", High Frequency Electronics, 2008.

20. Maci S, Borselli L. Diffraction at the edge of a truncated grounded dielectric slab. IEEE Transactions on Antennas and Propagation 1996; 44(6):863-873.

21. Abubeker A. Yussuf, Selcuk Paker, "Design of Wideband MIMO Antenna for Wireless Applications", 978-1-5090-64946/17/\$31.00 @2017 IEEE. 